\title{
Performance Evaluation of Energy Detection in Spectrum Sensing on the Cognitive Radio Networks
}

Rayan Abdelazeem Haboub Suliman ${ }^{1 *}$, Khalid Hamid Bilal ${ }^{2}$ and Ibrahim Elemam ${ }^{3}$

${ }^{1}$ Department of Engineering, Faculty of post graduate, Al Neelain University, Khartoum, Sudan

${ }^{2}$ Department of Communication, Faculty of engineering/university of science and technology, Khartoum, Sudan

${ }^{3}$ Department of Communication, El NeelainUniversity, Khartoum, Sudan

\begin{abstract}
Cognitive radio technology is a modernistic technology by which the idle licensed spectrum can be used by an unlicensed user which are called cognitive radio $\mathrm{CR}$ or secondary users. The CR technology was found to overcome the spectrum incompetence and inefficiency usage troubles. The major motivation of CR technology is current heavily underutilized of spectrum. Surly the primary user PU had all rights to use his spectrum band so CR mustn't interfere it on that band. Here we have to discuss about energy detection which is one of spectrum sensing techniques in CR functions and how to obtain the best available spectrum, to detect the spectrum hole, to estimate the optimum threshold voltage that produce the minimum Probability of false alarm and finally to enhance energy detection sensing algorithm by using MATLAB simulation.
\end{abstract}

Keywords: Cognitive radio; Energy detection; Threshold; Probability of false alarm

\section{Introduction}

Cognitive radio is an a enhanced to software-defined radio (SDR) that its automatically detects the surrounding RF, catalysts and smartly accommodates its operating parameters to the infrastructure of network according to meet user demand [1], if this band is further used by a licensed user, the cognitive radio stirs to other spectrum band or remains in the same band with altering its level of the transmission power or modulation scheme all of that avert interference, calibrations the congestion due to spectrum participating [2].

The unemployed spectrum was called white space or spectrum holes. The CRN is development of Dynamic Spectrum Access DSA and SDR by enabling the spectrum smartly and highly configuring radio transmitter/receiver.

There are four functions of CRN spectrum sensing, spectrum decision, spectrum management and spectrum sharing.

- Spectrum sensing: A CR user can occupy only the vacant spectrum band. Therefore a CR user should superintend the available bands spectrum, pick up their information, and therefore it can check the white space [1].

- Spectrum decision: Depend on the availability of spectrum on internal (and possibly external) policies [3], CR users can allocate a spectrum band.

- Spectrum sharing: Regard to there may be multiple cognitive radio users all of them are trying to access the spectrum, access on CRN should be arranged to prevent multiple users clash in interference bands of the spectrum [4].

- Spectrum mobility: Cognitive radio users are considered as guests on the spectrum. So if the particular servant spectrum band is wished for the primary user, the communication has to be continued in another idle spectrum band [5].

The spectrum sensing is the important one cause the CR technology are depend on it by which the spectrum holes are sensed [6], and it enables CR to configure to the environment, according to this point one of spectrum sensing techniques had taken to discuss and simulate on this paper.

\section{Spectrum Sensing Review}

It is a necessary issue of CR is to sense the spectrum holes, it's based on to be aware of and sensitive to changes on it surrounded environment [1], spectrum sensing algorithms enable the CR to adjust and adapt to the environment by detecting PUs which receiving data across the communication scope of CR users [7].

The sensing on CRN has two-main points:

a) To ensure that the secondary user doesn't caused interference to a primary user

b) To aid secondary user to recognize and utilize the white space for the required quality of service [2].

The spectrum sensing is to decide and determined between two hypotheses which are:

$$
\begin{aligned}
& x(t)=w(t), H 0(\text { PU absent) } \\
& x(t)=h n(t)+w(t), \text { H1 (PU present) }
\end{aligned}
$$

Where $\mathrm{x}(\mathrm{t})$ is the received signal by the unlicensed user, $\mathrm{n}(\mathrm{t})$ is the transmitted signal of the licensed user, $\mathrm{w}(\mathrm{t})$ is the Additive White Gaussian Noise (AWGN), h is the amplitude gain of the channel. H0 is a null hypothesis, means that there is no signal from licensed user [2], according to the primary user these hypotheses means:

H0: The spectrum band is idle, H1: The spectrum band is occupied [2].

The used sensing cognitive radio algorithm should be sensitive enough to characterize between the noise power and the signal power [7]. The main objective of this paper is to explicate and simulate specific

*Corresponding author: Rayan Abdelazeem Haboub Suliman, Department of Engineering, Faculty of post graduate, Al Neelain university, Khartoum, Sudan Tel: 0024912547363; E-mail: rere.abdoo2014@gmail.com

Received June 07, 2017; Accepted June 26, 2017; Published June 28, 2017

Citation: Suliman RAH, Bilal KH, Elemam I (2017) Performance Evaluation of Energy Detection in Spectrum Sensing on the Cognitive Radio Networks. J Electr Electron Syst 6: 228. doi: 10.4172/2332-0796.1000228

Copyright: (c) 2017 Suliman RAH, et al. This is an open-access article distributed under the terms of the Creative Commons Attribution License, which permits unrestricted use, distribution, and reproduction in any medium, provided the original author and source are credited. 
spectrum sensing algorithm and improve the performance of it for a low SNR environment.

As spectrum sensing is the key enabling in cognitive radio to adapt it is environment by detecting spectrum holes, so it could have categorized to three main types transmitter detection or noncooperative sensing, cooperative sensing and interference based sensing. Transmitter detection technique is further classified into matched filter detection, cyclostationary feature detection and energy detection which it discussed in this paper.

\section{Energy Detection}

It unveils the basic signal based on the sensed energy. According to its artlessness and no need to a prior knowledge of the signal of the primary user, energy detection (ED) is the most popular sensing technique [6]. Figure 1 shows the block diagram of ED technique.

In this method, signal is passed through band pass filter of the bandwidth $\mathrm{W}$ and is integrated over time interval, the output of the block of an integrator is then compared to a predefined threshold. The goal of this comparison is to discover the existence or absence of the licensed user. The value of threshold can set to be variable or fixed based on the conditions of the channel [6]. The Blind signal detector is the another name of ED [2], because it ignores the signal structure i.e., it estimates the presence of the signal by comparing the energy received with a known threshold $v$ derived from the statistics of the noise [8], analytically signal can be formalized as a hypothesis test

$$
\begin{aligned}
& \mathrm{Y}(\mathrm{k})=\mathrm{n}(\mathrm{k}) \ldots . H 0 \\
& \mathrm{Y}(\mathrm{k})=\mathrm{h}^{\star} \mathrm{s}(\mathrm{k})+\mathrm{n}(\mathrm{k}) \ldots . H 1
\end{aligned}
$$

In sample the $y(k)$ is analyzed at each instant $k$ and $n(k)$ is the noise of variance $\sigma^{2}$, say $y(k)$ be as sequence of received samples $k \in\{1,2 \ldots . N\}$ at the signal detector, therefore a decisionrule can be stated as

$$
\begin{aligned}
& \text { H0....if } \varepsilon<v \\
& \text { H1....if } \varepsilon>
\end{aligned}
$$

Where $\varepsilon=|\operatorname{Ey}(\mathrm{k})|^{2}$ the estimated energy of the received signal and $\mathrm{v}$ is is chosen to be the noise variance.

\section{Descriptive Analysis of ED}

The spectrum sensing algorithms are care about sensing the spectrum holes, these holes are immigrating with time and frequency so the spectrum holes detection should be real time. Spectrum sensing have a high computational and it needs to implement it a special hardware, furthermore the detection of these holes are affected by low SNR, so the used spectrum sensing algorithm should be able to distinguish the signal power from the noise power [2]. The ED method calculate the input signal energy and compares it with a threshold energy value, if the signal energy exceeds these threshold value the signal is presented in this particular frequency otherwise the frequency is empty [6].

The main objective is to determine the presence or the absence of a signal in a period of time. The spectrum of the input signal is computed

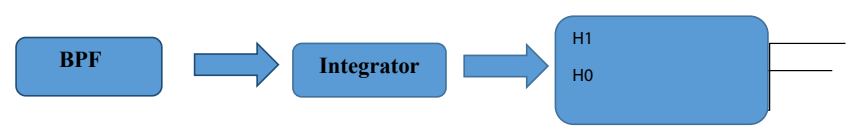

Figure 1: Energy detector block diagram. using eqn. 1 where $\mathrm{X}(\omega)$ is the Fourier transform of the input signal and $\mathrm{t} 2$ - $\mathrm{t} 1$ is the time period over which the input time samples are observed.

The threshold voltage is given by the expression in eqn. 3.1. The threshold voltage is related to the probability of false alarm and the noise power. The threshold energy spectrum given in the eqn. 5 can be computed by the following method.

The threshold value is defined by the classical Radar detection theory which limitation a threshold voltage [9] .For the input signal the energy spectrum of the signal and the energy spectrum of the threshold had calculated firstly to calculate the energy spectrum of the signal using energy spectrum computation mentioned in eqn. 5, secondly the energy spectrum of the threshold had to calculate by using calculation of threshold and threshold energy spectrum in eqns. 5-7. So the comparison had done between energy of the threshold and energy of the signal [9], if the energy of the threshold is greater than the energy of the signal that implies to presence of the frequency holes and the cognitive radio user can use it, otherwise there is no frequency holes at all [10].

All of that done based on Principal component analysis (PCA) which is a mathematical technique to minimize dimensionality of data.

\section{Mathematical Model}

$$
\begin{aligned}
& \text { Energy spectrum }=|\mathrm{x}(\omega)|^{2}\left(t_{2}-t_{1}\right) \\
& \mathrm{V}_{\mathrm{T}=\sqrt{2 \sigma_{\mathrm{n}}^{2} \log \left(\frac{1}{\mathrm{P}_{\mathrm{fa}}}\right)}} \mathrm{V}_{\mathrm{T}}(\omega)=\int_{\mathrm{t}_{1}}^{\mathrm{t}_{2}} \mathrm{~V}_{\mathrm{T}} \mathrm{e}^{-\mathrm{j} \omega \mathrm{t}} d t \\
& =\mathrm{V}_{\mathrm{T}}\left[\frac{\mathrm{e}^{-\mathrm{j} \omega \mathrm{t}}}{-\mathrm{j} \omega}\right]_{\mathrm{t}_{1}}^{\mathrm{t}_{2}} \\
& =j \frac{\mathrm{V}_{\mathrm{T}}}{\omega}\left[\mathrm{e}^{-\mathrm{j} \omega \mathrm{t}}\right]_{\mathrm{t}_{1}}^{\mathrm{t}_{2}} \\
& =\frac{\mathrm{V}_{\mathrm{T}}}{\omega} \mathrm{e}^{\mathrm{j}\left[\frac{\pi}{2}-\omega\left(\mathrm{t}_{2}-\mathrm{t}_{1}\right)\right]} \ldots . \\
& \left|\mathrm{V}_{\mathrm{T}}(\omega)\right|^{2}=\frac{\mathrm{V}^{2}}{\omega^{2}}\left(\mathrm{t}_{2}-\mathrm{t}_{1}\right) \\
& \mathrm{P}_{\mathrm{fa}}=10 \frac{\mathrm{V}_{\mathrm{T}}^{2}}{2 \sigma_{\mathrm{n}}^{2}}
\end{aligned}
$$

Where:

$\sigma_{\mathrm{n}}^{2}=$ Noise Power

$\mathrm{V}_{\mathrm{T}}=$ Threshold Voltage

$\omega=$ Frequency

$\mathrm{P}_{\mathrm{fa}}=$ Probability of False Alarm

$$
\begin{aligned}
& X(n)=\left[x_{1} \ldots x_{M} x_{M+1} \ldots x_{2 M} \ldots x_{M n}\right] \\
& L(n)=\left[x_{1} x_{2} \ldots x_{M}\right] \\
& L(n)=\left[x_{M+1} x_{M+2} \ldots x_{2 M}\right]^{T}
\end{aligned}
$$




$$
\begin{aligned}
& \mathrm{LL}_{\mathrm{i}}=\mathrm{L}(\mathrm{i}) \times \mathrm{L}^{\mathrm{T}}(\mathrm{i}) \\
& \mathrm{W}_{\mathrm{i}}=\sum_{i=1}^{n} \mathrm{LL}_{\mathrm{i}} \\
& \mathrm{W}_{\mathrm{x} . \mathrm{N}} \mathrm{E}_{\mathrm{j}}=\mathrm{Y}_{\mathrm{j}} \mathrm{E}_{\mathrm{j}} ; \mathrm{j}=1 \text { to } \mathrm{M} \\
& \text { Signal power }=\frac{1}{\mathrm{P}} \sum_{i=1}^{P} \mathrm{Y}_{\mathrm{i}} \\
& \text { Noise power }=\frac{1}{\mathrm{M}-\mathrm{P}} \sum_{i=P+1}^{M} \mathrm{Y}_{\mathrm{i}}
\end{aligned}
$$

\begin{tabular}{|c|}
\hline \multicolumn{2}{|c|}{ Input Signal } \\
\hline $\mathrm{X}(\mathrm{t}) \downarrow$ \\
Fourier Transform \\
$X(\dot{\omega})=\int x(t) e^{-j w t} \mathrm{dt}$ \\
\hline $\mathrm{x}(\dot{\omega}) \downarrow$ \\
\hline Power Spectrum \\
Power Spectrum $=\left|x(\dot{\omega})^{2}\right|$ \\
\hline Energy Spectrum \\
Energy Spectrum $=\left|x(\dot{\omega})^{2}\right|\left(t_{2}-t_{1}\right)$ \\
\hline
\end{tabular}

Figure 2: Flow for energy spectrum computation.

\section{Computer Model}

- Flow for energy spectrum computation (Figure 2).

- Conventional energy detection method (Figure 3).

\section{Simulation Environment}

Simulation environment is set as given in Table 1.

The simulation code had implemented Using MATLAB to find the relationship between Signals to Noise Ratio and Probability of False Alarm PFA for energy detection algorithm. Also the relationship among power of signal in Watt and signal to noise ratio in $\mathrm{dB}$ had implemented by using PCA technique.

\section{Results}

After execution of simulation code the results explained in form of graphs as follows:

Plot of Probability of False Alarm versus Signal to Noise Ratio for Energy Detection (Sensing Algorithm) at Vth=4 (Figure 4).

Plot of Probability of False Alarm versus Signal to Noise Ratio for Energy Detection (Sensing Algorithm) at Vth=8 (Figure 5).

\begin{tabular}{|l|c|}
\hline Parameters & Values \\
\hline Noise power $(\mathrm{np})$ & $0.1,0.2,0.3,0.4,0.5,0.6,0.7,0.8,0.9,1$ \\
\hline Energy of threshold (evt) & - \\
\hline Input signal $(\mathrm{xt})$ & Random \\
\hline Energy of the signal (ext) & - \\
\hline Threshold (VTh) & $4,8,12,16,18$ \\
\hline
\end{tabular}

Table 1: Simulation environment.

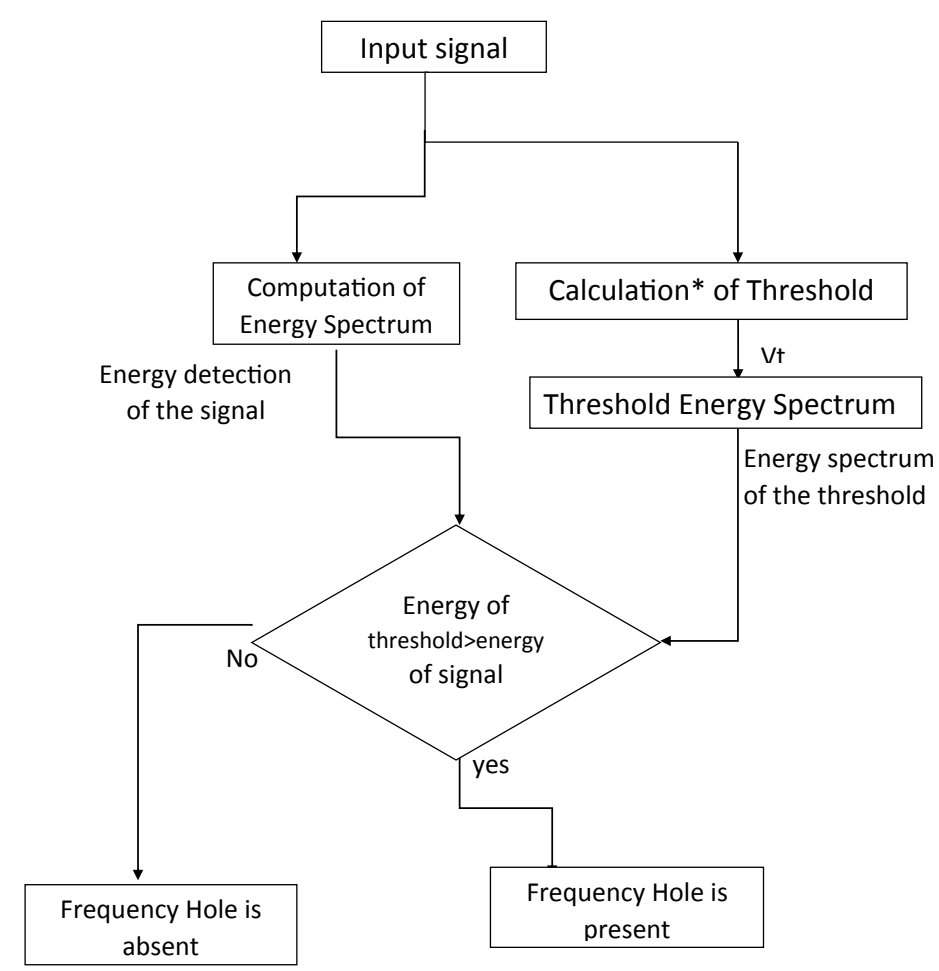

Figure 3: Conventional energy detection method. 
Citation: Suliman RAH, Bilal KH, Elemam I (2017) Performance Evaluation of Energy Detection in Spectrum Sensing on the Cognitive Radio Networks. J Electr Electron Syst 6: 228. doi: 10.4172/2332-0796.1000228

Page 4 of 5

Plot of Probability of False Alarm versus Signal to Noise Ratio for Energy Detection (Sensing Algorithm) at Vth=12 (Figure 6).

Plot of Probability of False Alarm versus Signal to Noise Ratio for Energy Detection (Sensing Algorithm) at Vth=18 (Figure 7).

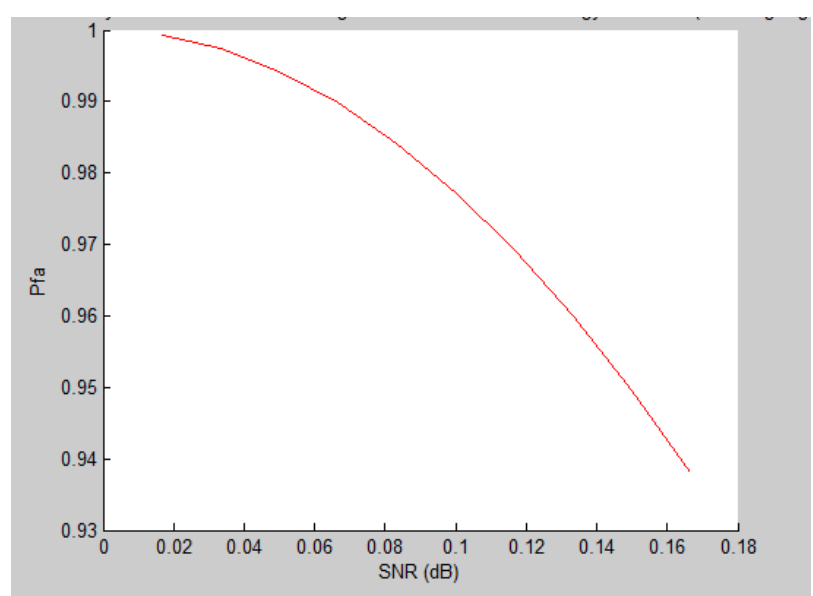

Figure 4: Plot of Probability of False Alarm versus Signal to Noise Ratio for Energy Detection (Sensing Algorithm).

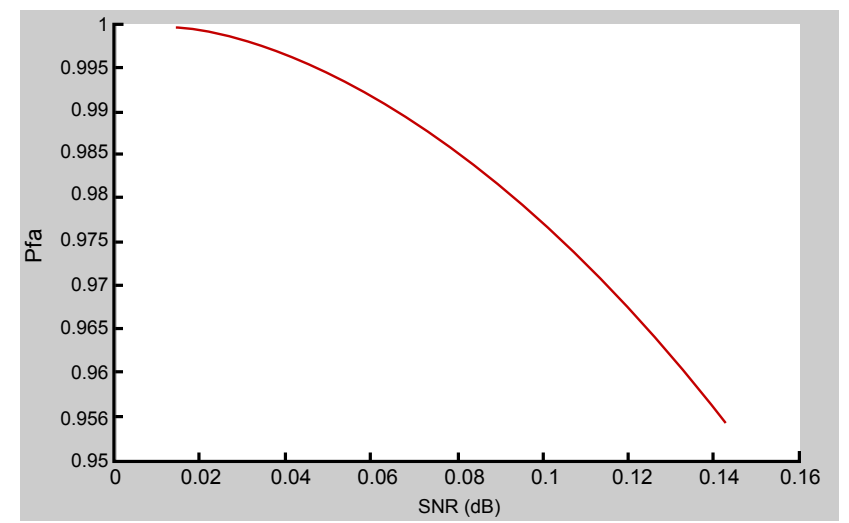

Figure 5: Plot of Probability of False Alarm versus Signal to Noise Ratio for Energy Detection (Sensing Algorithm).

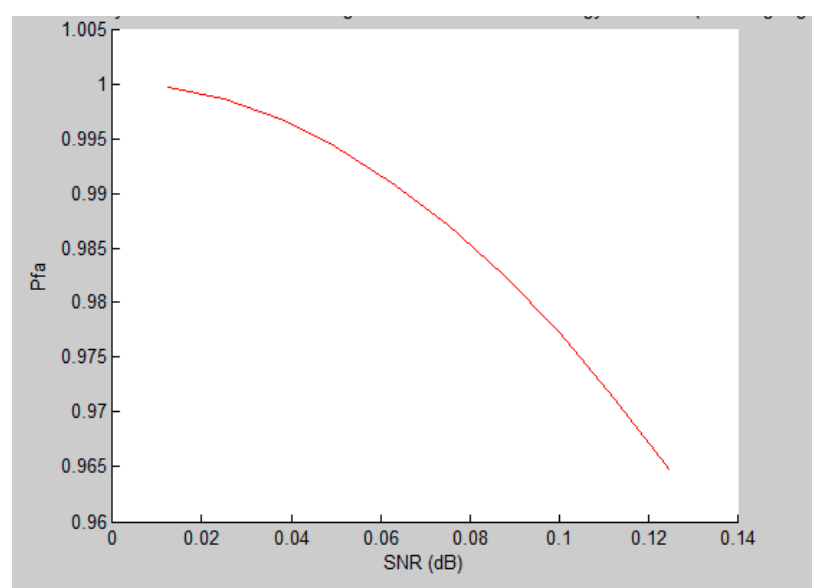

Figure 6: Plot of Probability of False Alarm versus Signal to Noise Ratio for Energy Detection (Sensing Algorithm).
Plot of Power in Watts vs. SNR in dB for Energy Detection using PCA Technique (Figures 8 and 9).

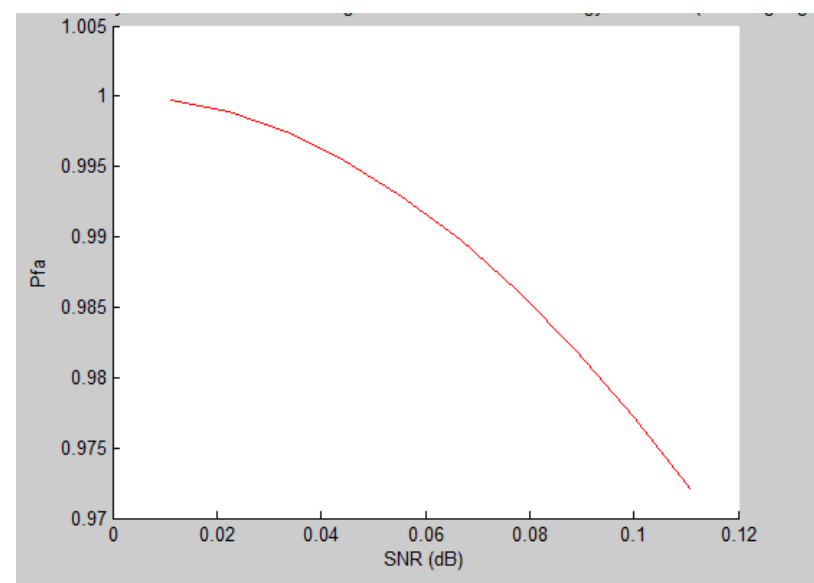

Figure 7: Plot of Probability of False Alarm versus Signal to Noise Ratio for Energy Detection (Sensing Algorithm).

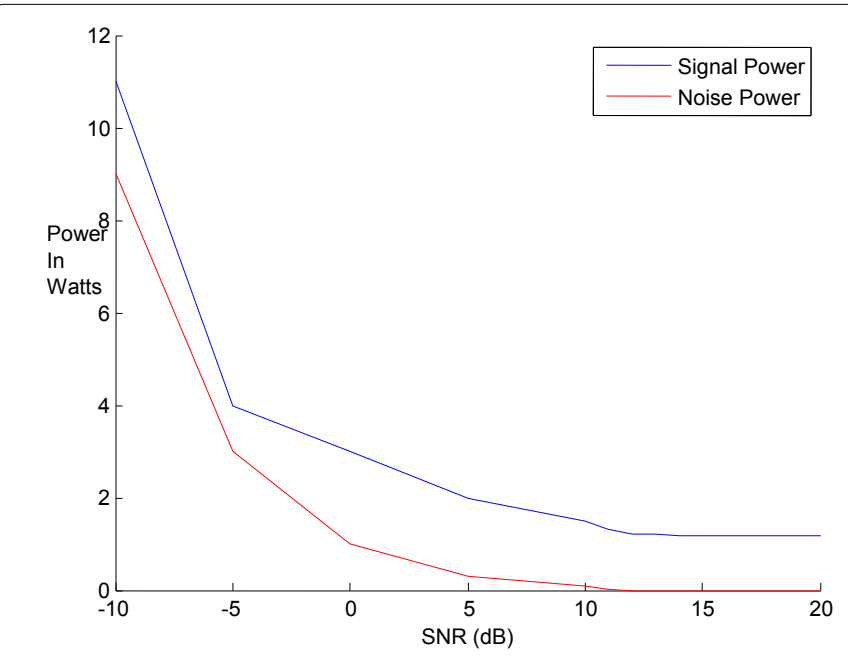

Figure 8: Plot of Power in Watts Vs SNR in $\mathrm{dB}$ for Energy Detection Using PCA Technique.

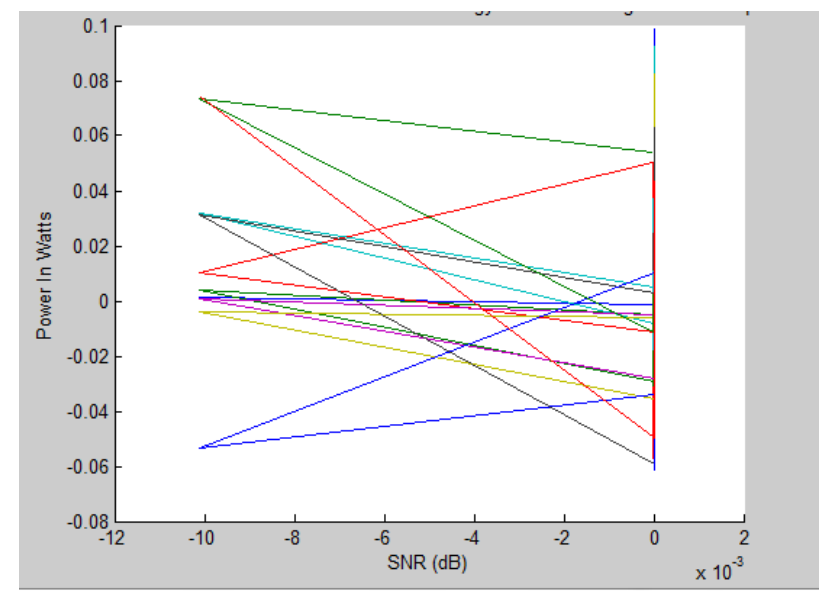

Figure 9: Plot of power in Watts Vs SNR in dB for Energy Detection Using PCA Technique by random data entry ranges. 
Citation: Suliman RAH, Bilal KH, Elemam I (2017) Performance Evaluation of Energy Detection in Spectrum Sensing on the Cognitive Radio Networks. J Electr Electron Syst 6: 228. doi: 10.4172/2332-0796.1000228

Page 5 of 5

\section{Results Discussion}

From eqns. 5-7, the signal to noise ratio and probability of false alarm are calculated and the relationship between them is plotted in Figures 4-7. From graphs the relationship between the signal to noise ratio (SNR) and probability of false alarm (Pfa) is found to be as follows:

SNR is reversely proportional to pfa, i.e., as SNR increases Pfa decreases when threshold (VTh) is constant for the varying values of noise Power from 0.1 up to 1 (step 0.1). The predefined range of threshold is between 4 and 18, the smallest values of (SNR) is found to be when using (VTh=18 which is max of Vth), then the (Pfa) is proportional to (VTh).

\section{Conclusion}

Cognitive radio technology is a modernistic technology by which the idle licensed spectrum can be used by an unlicensed user which is called cognitive radio CR. The CR technology was found to overcome the spectrum incompetence and inefficiency usage troubles. The major motivation of CR technology is current heavily underutilized of spectrum. Surly the PU have all rights to use his spectrum band so CR mustn't interfere it on that band. This paper discussed about energy detection which is one of spectrum sensing techniques, explained how to obtain the best available spectrum, to detect the spectrum hole, to estimate the optimum Threshold voltage that produce the minimum Probability of false alarm, calculate the power of signal in special range and random one and finally to enhance Energy Detection Sensing Algorithm.

\section{References}

1. Yucek T, Arslan $H$ (2009) A survey of spectrum sensing algorithms for cognitive radio applications. IEEE Communications Surveys and Tutorials 11: 116-130.

2. Lassila P, Penttinen A (2009) Survey on performance analysis of cognitive radio networks. COMNET Department, Helsinki University of Technology, P.O Box 3000, FIN 02015 TKK, Finland.

3. Kaur MJ, Uddin M, Verma HK (2010) Analysis of Decision Making Operation in Cognitive radio using Fuzzy Logic System. India International Journal of Computer Applications 4: 35-39.

4. Hou YT, Shi Y, Sherali HD (2008) Spectrum sharing for multi-hop networking with cognitive radios. IEEE Journal on Selected Areas in Communications 26 146-155.

5. Verma S, Chawla M (2015) A Survey on Spectrum Mobility in Cognitive Radio Network. International Journal of Computer Applications 119: 33-36.

6. Haykin S (2005) Cognitive Radio: Brain-Empowered Wireless Communications. IEEE Journal on Selected Areas in Communications 23: 201-220.

7. Ghasemi A, Sousa ES (2005) Collaborative Spectrum Sensing for Opportunistic Access in Fading Environment. IEEE DySPAN, pp: 131-136.

8. Ghasemi A, Sousa ES (2008) Spectrum sensing in cognitive radio networks requirements, challenges and design trade-offs. IEEE Communications Magazine 46: 32-39.

9. Matinmikko M, Mustonen M, Sarvanko H, Höyhtyä M, Hekkala A, et al. (2008) A Motivating Overview of Cognitive Radio: Foundations, Regulatory Issues and Key Concepts. First International Workshop CogART, Aalborg.

10. Chincholkar AA, Thakare $\mathrm{CH}$ (2014) Matlab implementation of spectrum sensing methods in cognitive radio. Global Journal of Engineering Science and Research Management, pp: 19-28. 